\title{
On Singular Sturm-Liouville Problem with Impulse
}

\author{
Rauf Kh AMİROV, Selma GÜLYAZ \\ Department of Mathematics, Faculty of Science, Cumhuriyet University, 58140 Sivas, Turkey \\ Email: amirovrauf@gmail.com, sgulyaz@cumhuriyet.edu.tr
}

\begin{abstract}
Sturm-Liouville problem with boundary and discontinuity conditions was studied. For the solution inverse problem necessary and sufficient condition was obtained by the classical Gelfand-Levitan-Marchenko (GLM) type main integral equation and also algorithm was already given.
\end{abstract}

Keywords: Impulse conditions, Inverse problem, Kernel, Integral equation

\section{Introduction}

We consider boundary value problem $L$ for the equation:

$$
l(y):=-y^{\prime \prime}+q(x) y=\lambda y, \lambda=k^{2}, 0<x<\pi
$$

on the interval $0<x<\pi$ with the boundary conditions

$$
U(y):=y^{\prime}(0)=0, V(y):=y(\pi)=0
$$

and with the jump conditions

$$
y(d+0)=a y(d-0), y^{\prime}(d+0)=a^{-1} y^{\prime}(d-0),
$$

where $\lambda$ is the spectral parameter, $q(x)$ is a real valued function with $q(x) \in L_{2}(0, \pi)$ and $a(a>0, a \neq 1)$ is a real constant, $d \in\left(0, \frac{\pi}{2}\right)$.

Inverse spectral analysis has been an important research topic in mathematical physics. Inverse problems of spectral analysis involve the reconstruction of a linear operator from its spectral characteristics e.g., see $[2,8,14,20]$. Later, inverse problems for a regular and singular Sturm- Liouville operator appeared in various versions $[4,10,12,14,16,17,19,21,22]$.

Assuming that heat flows only into the liquid which has an uninform density $\rho(x)$ and is convected only from the liquid into the surrounding medium, the initial boundary value problem for a bar of length one takes the form

$$
\begin{gathered}
u_{t}=\rho(x) u_{x x} \\
u_{x}(0, t)=0 \\
-k A u_{x}(\pi, t)=Q M(d v / d t)+k_{1} B v(t) \text { for all } t, \\
u(x, 0)=u_{0}(x) \text { for } x \in[0, \pi] \\
v(0)=v_{0}
\end{gathered}
$$

after factoring out the steady-state solution, where

$$
\rho(x)=\left\{\begin{array}{c}
1, \quad 0<x<d \\
\alpha^{2}, d<x<\pi
\end{array}\right.
$$

Assuming that the rate of heat transfer across the liquid solid interface is proportional to the difference in temperature between the end of the bar and the liquid with which it is in contact (Newton's law of cooling) and applying Fourier's law of heat conduction at $x=\pi$, we get 
$v(t)=u(\pi, t)+k c^{-1} u_{x}\left(\pi^{-1}, t\right)$ for $t>0$, where $c>0$ is the coefficient of heat transfer for the liquid. If we put $u(x, t)=y(x) \exp (-\lambda t)$, then problems (1.1)-(1.3) will appear to be the result of the above problem. Indeed, condition (1.2) is obtained from $\left(3^{*}\right)$, easily. Here

$$
H=\frac{c}{k}, \quad H_{1}=\frac{c A+k_{1} B}{Q M} \text { and } \quad H_{2}=\frac{k_{1} B c}{Q M_{k}} .
$$

Finally, if we put

$$
t=\left\{\begin{array}{c}
x, \quad 0<x<d, \\
\alpha x, d<x<\pi,
\end{array}\right.
$$

then the discontinuity conditions (1.3) and a particular case of equation (1.1) will appear. This corresponds to the case of nonperfect thermal contact. Since the density is changed at one point in the interval, both the intensity and the instant velocity of heat change at this point. Hence, equation (1.1)-(1.3) will appear to be the result of the above problem.

Boundary value problems with discontinuity conditions inside the interval often appear in applications. Such problems are connected with discontinuous material properties. Inverse problems with a discontinuity condition inside the interval frequently arise in mathematics, mechanics, radio electronics, geophysics, and other fields of science and technology. For example, discontinuous inverse problems appear in electronics for constructing parameters of heterogeneous electronic lines with desirable technical characteristics [15, 18]. As a rule, such problems are related to discontinuous and nonsmooth properties of a medium (e.g., see $[10,13])$. Discontinuous inverse problems (in various formulations) have been considered in [10, 13] and other works. Generally, for recovering the potential function on the whole interval it is necessary to specify two spectra of boundary value problems with different boundary conditions. The inverse problem for interior spectral data of the differential operator consists in reconstruction of this operator from the known eigenvalues and some information on eigenfunctions at some internal point.

A complete solution of the inverse spectral problem for a class of Sturm-Liouville operators must consist of two parts: (1) an explicit description of the set of spectral data for the operators in Sturm-Liouville and (2) development and justification of the method of recovering the operator in Sturm-Liouville that corresponds to arbitrary given spectral data in spectral data. The algorithm of recovering the potential $\mathrm{q}$ from the spectral data of a regular Sturm-Liouville operator based on the transformation operators and the so-called Gelfand-Levitan-Marchenko equation was developed by Gelfand-Levitan [8] and Marchenko [16] in early 1950-ies.

The first complete solution of the inverse problem that is based on an exact integral approach was obtained by Gelfand and Levitan $[1,8]$ for the potential problem in the Schrodinger wave equation. In electro magnetics, the above approach is directly applicable to the case of inversion with a transient plane wave, normally incident on a planar stratified lossless medium [9], provided that the wave equation is converted to the Schrodinger equation. Generalizations of the Gelfand-Levitan approach to the case of oblique incidence [5], dissipative media [12], etc, were all based on deriving a Schrodinger-type equation from the basic wave equation through a series of transformations, and reconstructing the unknown potential, which is related to the medium parameters, via the Gelfand-Levitan procedure. Other inverse methods which are based on an integral equation and are in the same spirit as the Gelfand-Levitan approach are the ones due to [9]. A review of some of these integral inverse methods and others can be found in the review paper by Newton [28].

Faydaoglu and Guseinov [7] had considered the following eigenvalue problems of differential equations with impulsive perturbation

$$
\begin{gathered}
-\left[p(t) x^{\prime}\right]^{\prime}+q(t) x=\nu \rho(t) x, t \in[0, a) \cup(a, b] \\
x\left(a^{-}\right)=\alpha x\left(a^{+}\right), x^{\prime}\left(a^{-}\right)=\beta x^{\prime}\left(a^{+}\right) \\
x(0)=x(b)=0 .
\end{gathered}
$$

They proved that the eigenvalue problem $\left(5^{*}\right)-\left(7^{*}\right)$ has a countably infinite set of eigenvalues $\nu_{0}, \nu_{2}, \ldots$ tending to $+\infty$ and eigenfunctions was proved and a uniformly convergent expansion formula in the eigenfunctions was established. 
In this aspect, the studies of Gelfand, Levitan [8], [14] and Marchenko [25] include basic investigations related to the integral representations of solutions to various direct and inverse problems for the SturmLiouville differential operators.

Inverse spectral problems were studied for the second-order differential operators on a finite interval with discontinuity conditions inside the interval.

As different from [21], in this study, solution of inverse problem is reduced to the solution of the Gelfand-Levitan-Marchenko (GLM) type main integral equation which is used for solution of inverse problem in classical case. Item 3 deals with the solution of the inverse problem. We prove existence and uniqueness of the solution of the Gelfand-Levitan-Marchenko (GLM) differential equation and give a procedure for the solvability of classical inverse problem for impulsive differential operators. Also, necessary and sufficient conditions for existence of solution of inverse problem are mentioned in terms of given sequences. Moreover, an example which contains an algorithm for solution of inverse problem is given in the end of this paper.

\section{Preliminaries}

Let the function $\varphi(x, \lambda)$ be the solution of equation (1.1) that satisfies the initial conditions

$$
\varphi(0, \lambda)=1, \varphi^{\prime}(0, \lambda)=0
$$

and the jump condition (1.3). Let $\lambda_{0}, \lambda_{1}, \ldots$ be the eigenvalues of the boundary value problem (1.1)-(1.3). Then $\varphi\left(x, \lambda_{n}\right)(n \geq 0)$ are the eigenfunctions of this boundary value problem. Let $\varphi_{0}\left(x, \lambda_{n}^{0}\right)(n \geq 0)$ be a solution of the equation (1.1) in the case $q(x)=0$ satisfying the conditions (1.2)-(1.3). $\lambda_{0}^{0}, \lambda_{1}^{0}, \ldots$ are eigenvalues of the boundary value problem (1.1)-(1.3) when $q(x)=0$. The numbers $\alpha_{n}$ which

$$
\alpha_{n}=\int_{0}^{\pi} \varphi^{2}\left(x, \lambda_{n}\right) d x, n=0,1, \ldots
$$

are called the normalizing constant of the boundary value problem (1.1)-(1.3) .

Then, the numbers $\alpha_{n}^{0},(n=0,1, \ldots)$ are called the normalizing constant of the boundary value problem (1.1)-(1.3) when $q(x)=0$.

It is easy that the functions $e_{0}(x, \lambda)$ is the solution of equation (1.1) in the case $q(x) \equiv 0$ satisfying the initial conditions $e_{0}(0, \lambda)=1, e_{0}^{\prime}(0, \lambda)=i k$ and the jump conditions (1.3) can be written as

$$
e_{0}(x, \lambda)= \begin{cases}e^{i k x}, & 0<x<d, \\ a^{+} e^{i k x}+a^{-} e^{i k(2 d-x)}, & d<x<\pi\end{cases}
$$

where $a^{ \pm}=\frac{1}{2}\left(a \pm \frac{1}{a}\right)$.

The following theorem related to the integral representation (transformation operator) for the solution $e(x, \lambda)$ can be found in [4].

Theorem 1. [4, Theorem 1.] Let $\int_{0}^{\pi}|q(t)| d t<+\infty$. Then each solution satisfying the initial conditions $e_{0}(0, \lambda)=1, e_{0}^{\prime}(0, \lambda)=i k$ and the jump conditions (1.3) has the form

$$
e(x, \lambda)=e_{0}(x, \lambda)+\int_{-x}^{x} K(x, t) e^{i k t} d t
$$

with $\int_{-x}^{x}|K(x, t)| d t \leq e^{c \sigma_{1}(x)}-1$ where $\sigma_{1}(x)=\int_{0}^{x}(x-t)|q(t)| d t, c=a^{+}+\left|a^{-}\right|+1$.

If the function $q(x)$ is differentiable then the kernel $K(x, t)$ satisfies the following properties: 


$$
\begin{aligned}
& \widetilde{K}_{x x}(x, t)-q(x) \widetilde{K}(x, t)=\widetilde{K}_{t t}(x, t), \quad \widetilde{K}(x, x)=\frac{a^{+}}{2} \int_{0}^{x} q(t) d t, \\
& \widetilde{K}(x, 2 d-x+0)-\widetilde{K}(x, 2 d-x-0)=\frac{a^{-}}{2} \int_{0}^{x} q(t) d t, \\
& \widetilde{K}(x,-x)=0 \text { where } \widetilde{K}=K(x, t)+K(x,-t) .
\end{aligned}
$$

Remark 1. [4, Remark] It is easily shown that if $q(x) \in L_{2}[0, \pi]$ then $K_{x}(x, \cdot) \in L_{2}[0, \pi]$ and $K_{t}(x, \cdot) \in L_{2}[0, \pi]$.

Let us denote problem $L$ as $L_{0}$ in the case of $q(x) \equiv 0$. It is easily shown that the solution $\varphi_{0}(x, k)$ satisfying the initial conditions $\varphi_{0}(0, k)=1, \varphi_{0}^{\prime}(0, k)=0$ and the jump conditions (1.3) can be written as

$$
\varphi_{0}(x, \lambda)=\left\{\begin{array}{lr}
\cos k x, & 0<x<d, \\
a^{+} \cos k x+a^{-} \cos k(2 d-x), & d<x<\pi,
\end{array}\right.
$$

Let $\Delta_{0}(k)$ be a characteristic function of problem $L_{0}$. Then characteristic equation of the problem $L_{0}$ is written as

$$
\Delta_{0}(k) \equiv a^{+} \cos k \pi+a^{-} \cos k(2 d-\pi)=0 .
$$

The roots $k_{n}^{0}$ of this equation are eigenvalues of the problem $L_{0}$. Under these conditions boundary value problem (1.1)-(1.5)possesses the following spectral properties:

a) $\inf _{n \neq m}\left|k_{n}^{0}-k_{m}^{0}\right|=\beta>0$, i.e., roots of characteristic equation $\Delta_{0}(k)=0$ are separated.

b) Eigenvalues of the problem $L$ are simple, that is $\dot{\Delta}\left(k_{n}\right) \neq 0$.

c) Eigenvalues of the problem $L$ have the following asymptotic behavior

$$
k_{n}=k_{n}^{0}+\frac{d_{n}}{k_{n}^{0}}+\frac{\delta_{n}}{k_{n}^{0}},
$$

where $\delta_{n}=\frac{1}{k_{n}^{0}} \int_{0}^{\pi} K_{t}^{\prime}(\pi, t) \sin k_{n}^{0} t d t \in \ell_{2}, k_{n}^{0}=n+h_{n}, \sup \left|h_{n}\right|<+\infty$ and

$d_{n}=\frac{a^{+} \sin k_{n}^{0} \pi-a^{-} \sin k_{n}^{0}(2 d-\pi)}{2 \dot{\Delta}_{0}\left(k_{n}^{0}\right) k_{n}^{0}} \int_{0}^{\pi} q(t) d t$ is a bounded sequence.

d) Normalizing numbers of the problem $L$ have the asymptotic behavior

$$
\alpha_{n}=\alpha_{n}^{0}+\frac{\delta_{n}}{n}
$$

where

$$
\alpha_{n}^{0}=\left(\left(a^{+}\right)^{2}+\left(a^{-}\right)^{2}\right) \frac{\pi-d}{2}+\frac{d}{2}+2 a^{+} a^{-}(\pi-d) \cos 2 k_{n}^{0} d+\delta_{1 n}
$$

and

$$
\begin{aligned}
& \delta_{1 n}=\frac{\sin 2 k_{n}^{0} d}{4 k_{n}^{0}}+\left(a^{+}\right)^{2} \frac{\sin 2 k_{n}^{0} \pi}{4 k_{n}^{0}}-\left(a^{+}\right)^{2} \frac{\sin 2 k_{n}^{0} d}{4 k_{n}^{0}}+\frac{a^{+} a^{-}}{k_{n}^{0}} \sin 2 k_{n}^{0}(\pi-d) \\
& -\frac{\left(a^{-}\right)^{2}}{4 k_{n}^{0}} \sin 2 k_{n}^{0}(2 d-\pi)+\frac{\left(a^{-}\right)^{2}}{4 k_{n}^{0}} \sin 2 k_{n}^{0} d, \delta_{n} \in \ell_{2} .
\end{aligned}
$$

Lemma 1. The specification of the spectrum $\left\{\lambda_{n}\right\}_{n \geq 0}$ uniquely determines the characteristic function $\Delta(\lambda)$ by the formula

$$
\Delta(\lambda)=\prod_{n=0}^{\infty} \frac{\lambda_{n}-\lambda}{\lambda_{n}^{0}}
$$


Proof. It follows from

$$
\Delta(\lambda) \equiv a^{+} \cos k \pi+a^{-} \cos k(2 d-\pi)+\int_{0}^{\pi} \tilde{K}(\pi, t) \cos k t d t
$$

that $\Delta(k)$ is entire in $\lambda$ of order $1 / 2$, and consequently by Hadamard's factorization theorem [6, p.289]. $\Delta(\lambda)$ is uniquely determined up to a multiplicative constant by its zero:

$$
\Delta(\lambda)=C \prod_{n=0}^{\infty}\left(1-\frac{\lambda}{\lambda_{n}}\right)
$$

consider the function. For $\lambda \rightarrow 0$ we obtain

$$
\Delta_{0}(\lambda) \equiv a^{+} \cos k \pi+a^{-} \cos k(2 d-\pi)=C_{0} \prod_{n=0}^{\infty}\left(1-\frac{\lambda}{\lambda_{n}^{0}}\right),
$$

where $C_{0}=a^{+}+a^{-}=a$. Then $\frac{\Delta(\lambda)}{\Delta_{0}(\lambda)}=\frac{C}{a} \prod_{n=0}^{\infty} \frac{\lambda_{n}^{0}}{\lambda_{n}} \prod_{n=0}^{\infty}\left(1+\frac{\lambda_{n}-\lambda_{n}^{0}}{\lambda_{n}^{0}-\lambda}\right)$.

Taking (2.4) and (2.8) into account we calculate

$$
\lim _{\lambda \rightarrow+i \infty} \frac{\Delta(\lambda)}{\Delta_{0}(\lambda)}=1, \lim _{\lambda \rightarrow+i \infty} \prod_{n=0}^{\infty}\left(1+\frac{\lambda_{n}-\lambda_{n}^{0}}{\lambda_{n}^{0}-\lambda}\right)=1 \text { and hence } C=a \prod_{n=0}^{\infty} \frac{\lambda_{n}}{\lambda_{n}^{0}} \text {. Substituting this }
$$

into (2.9) we arrive at (2.7).

When $q(x)=0$ formula of $\varphi_{0}\left(x, \lambda_{n}^{0}\right)$ is as follows;

$$
\varphi_{0}\left(x, k_{n}^{0}\right)=\left\{\begin{array}{lr}
\cos k_{n}^{0} x, & 0<x<d, \\
a^{+} \cos k_{n}^{0} x+a^{-} \cos k_{n}^{0}(2 d-x), & d<x<\pi .
\end{array}\right.
$$

One can consider the relation (2.3) with respect to $\cos k x$. Solving this equation we obtain

$$
\cos k x=\left\{\begin{array}{lr}
\varphi_{0}(x, k), & 0<x<d \\
a^{+} \varphi_{0}(x, k)-a^{-} \varphi_{0}(2 d-x, k), & d<x<\pi
\end{array}\right.
$$

\section{Results}

The Wronskian of any two solutions $y_{1}(x, \lambda)$ and $y_{2}(x, \lambda)$ of $(1.1)-(1.3)$ is constant on $[0, d)$ and $(d, \pi]$ and using the impulse conditions (1.3) we get

$\left.W\left(y_{1}, y_{2}\right)\right|_{x=d+0}=\left.W\left(y_{1}, y_{2}\right)\right|_{x=d-0}$.

Theorem 2. (i) The system of eigenfunctions $\left\{\varphi\left(x, k_{n}\right)\right\}_{n \geq 0}$ of the boundary value problem $L$ is complete in $L_{2}(0, \pi)$.

(ii) Let $f(x), x \in[0, d) \cup(d, \pi]$ be an absolutely continuous function and $f(d+0)=a f(d-0), f^{\prime}(d+0)=a^{-1} f^{\prime}(d-0)$. Then

$$
f(x)=\sum_{n=0}^{\infty} a_{n} \varphi\left(x, k_{n}\right), \quad a_{n}=\frac{1}{\alpha_{n}} \int_{0}^{\pi} f(t) \varphi\left(t, k_{n}\right) d t
$$

and the series converges uniformly on $[0, \pi]$.

(iii) For $f(x) \in L_{2}(0, \pi)$ the series (3.1) converges in $L_{2}(0, \pi)$ and

$$
\int_{0}^{\pi}|f(x)|^{2} d x=\sum_{n=0}^{\infty} \alpha_{n}\left|a_{n}\right|^{2}, \quad \text { (Parseval's equality) }
$$


Proof of this Theorem 2 can be done by using proof of Theorem in reference [2].

In addition, we obtain the theorem that the statement above, since the eigenfunctions $\left\{\varphi\left(x, k_{n}\right)\right\}_{n>0}$ are complete and orthogonal in $L_{2}(0, \pi)$, they form an orthogonal basis in $L_{2}(0, \pi)$ and Parseval's equality (3.2) is valid.

We will refer to the sequences $\left\{\lambda_{n}\right\}_{n>0}$ and $\left\{\alpha_{n}\right\}_{n \geq 0}$ as the spectral characteristics of the boundary value problem (1.1)-(1.3). Consider the function

$$
\begin{aligned}
F_{0}(x, t) & =\sum_{n=0}^{\infty}\left(\frac{\varphi_{0}\left(t, k_{n}\right) \cos k_{n} x}{\alpha_{n}}-\frac{\varphi_{0}\left(t, k_{n}^{0}\right) \cos k_{n}^{0} x}{\alpha_{n}^{0}}\right) \\
F(x, t)= & a^{+} F_{0}(x, t)+a^{-} F_{0}(2 d-x, t) \\
& =\sum_{n=0}^{\infty}\left(\frac{\varphi_{0}\left(t, k_{n}\right) \varphi_{0}\left(x, k_{n}\right)}{\alpha_{n}}-\frac{\varphi_{0}\left(t, k_{n}^{0}\right) \varphi_{0}\left(x, k_{n}^{0}\right)}{\alpha_{n}^{0}}\right)
\end{aligned}
$$

with the help $\left\{\lambda_{n}\right\}_{n \geq 0}$ and $\left\{\alpha_{n}\right\}_{n \geq 0}$ sequences.

Theorem 3. [15, Theorem 3] For each fixed $x \in(0, \pi]$, the kernel $\tilde{K}(x, t)$ appearing in representation

$$
\varphi(x, \lambda)=\varphi_{0}(x, \lambda)+\int_{0}^{x} \tilde{K}(x, t) \cos k t d t
$$

satisfies the linear integral equation

$$
F(x, t)+a^{+} \tilde{K}(x, t)-a^{-} \tilde{K}(x, 2 d-t)+\int_{0}^{x} \tilde{K}(x, \xi) F_{0}(\xi, t) d \xi=0, \quad 0<t<x .
$$

Theorem 4. For each fixed $x \in(0, \pi]$ equation (3.6) has a unique solution $\tilde{K}(x, \cdot) \in L_{2}(0, x)$.

Proof. Firstly, we show that (3.6) can be written such as $(I+B) f=g$ for $x>d$ where $B: L_{2}(0, \pi) \rightarrow$ $L_{2}(0, \pi)$ is linear bounded operator, and $I$ is identity operator. It is obvious that $(I+B) f=g$ for $x \leq d$.

For $x>d$, we write $(3.6)$ such as $L_{x} \tilde{K}(x, \cdot)+K_{x} \tilde{K}(x, \cdot)=-F(x, \cdot)$ where

$$
\begin{aligned}
& \left(L_{x} f\right)(t)= \begin{cases}f(t) & , t \leq d<x \\
a^{+} f(t)-a^{-} f(2 d-t) & , d<t<x\end{cases} \\
& \left(K_{x} f\right)(t)=\int_{0}^{x} f(\xi) F_{0}(\xi, t) d \xi, \quad 0<t<x .
\end{aligned}
$$

Now, we show that $L_{x}$ has a boundary inverse in $L_{2}(0, \pi)$. We have

$$
\left(L_{x} f\right)(t)=a^{+} f(t)-a^{-} f(2 d-t)=\varphi(t), \quad \varphi(t) \in L_{2}(0, \pi) .
$$

Substituting $2 d-t$ by $t$ in (3.7), we obtain that

$$
\varphi(2 d-t)=a^{+} f(2 d-t)-a^{-} f(t) .
$$

Subtract (3.8) from (3.7), we get

$$
\begin{aligned}
& f(t)=\left(L_{x}^{-1} \varphi\right)(t)= \begin{cases}\varphi(t) & , t \leq d<x \\
a^{+} \varphi(t)-a^{-} \varphi(2 d-t) & , d<t<x .\end{cases} \\
& \int_{0}^{\pi}|f(t)|^{2} d t=\int_{0}^{d-0}|f(t)|^{2} d t+\int_{d+0}^{\pi}|f(t)|^{2} d t \\
& =\int_{0}^{d-0}|\varphi(t)|^{2} d t+\int_{d+0}^{\pi}\left|a^{+} \varphi(t)-a^{-} \varphi(2 d-t)\right|^{2} d t \\
& \leq \int_{0}^{d-0}|\varphi(t)|^{2} d t+a^{+} \int_{d+0}^{\pi}|\varphi(t)|^{2} d t+a^{-} \int_{2 d-\pi}^{d-0}|\varphi(t)|^{2} d t \text {. }
\end{aligned}
$$


Since $\varphi(t)=0$ for $t>\pi$, we have

$\|f(t)\|_{L_{2}(0, \pi)}=\int_{0}^{\pi}|f(t)|^{2} d t \leq C \int_{0}^{\pi}|\varphi(t)|^{2} d t=C\|\varphi(t)\|_{L_{2}(0, \pi)}$. Hence, $L_{x}$ has boundary inverse in $L_{2}(0, \pi)$. Thus, we can write the main integral equation (3.6) as

$$
\tilde{K}(x, \cdot)+\left(L_{x}^{-1} K\right) \tilde{K}(x, \cdot)=-L_{x}^{-1} F(x, \cdot)
$$

where $L_{x}^{-1} K$ is completely continuous operator in $L_{2}(0, \pi)$. Since (3.6) is a Fredholm equation, it is sufficient to prove that the homogeneous equation

$$
a^{+} \tilde{K}(t)-a^{-} \tilde{K}(2 d-t)+\int_{0}^{x} \tilde{K}(\xi) F_{0}(\xi, t) d \xi=0
$$

has only the trivial solution $\tilde{K}(t)=0$ by [17, Theorem 3 ]. Let $\tilde{K}(t)$ be a solution of (3.9). Then $\int_{0}^{x}\left(a^{+} \tilde{K}(t)-a^{-} \tilde{K}(2 d-t)\right)^{2} d t$

$$
+\int_{0}^{x}\left(a^{+} \tilde{K}(t)-a^{-} \tilde{K}(2 d-t)\right) \int_{0}^{x} \tilde{K}(\xi) F_{0}(\xi, t) d \xi d t=0 .
$$

By using (2.11) and (3.3), also we consider that $\tilde{K}(\xi)=0$ for $\xi<2 d-x$, to get

$$
\begin{aligned}
& \int_{0}^{x}\left(a^{+} \tilde{K}(t)-a^{-} \tilde{K}(2 d-t)\right)^{2} d t+\left(a^{+}\right)^{2} \int_{0}^{x} \tilde{K}(t) \int_{d}^{x} \tilde{K}(\xi) \sum_{n=0}^{\infty} \frac{\varphi_{0}\left(t, k_{n}\right) \varphi_{0}\left(\xi, k_{n}\right)}{\alpha_{n}} d \xi d t \\
& -a^{+} a^{-} \int_{0}^{x} \tilde{K}(t) \int_{d}^{x} \tilde{K}(\xi) \sum_{n=0}^{\infty} \frac{\varphi_{0}\left(t, k_{n}\right) \varphi_{0}\left(2 d-\xi, k_{n}\right)}{\alpha_{n}} d \xi d t \\
& -a^{+} a^{-} \int_{0}^{x} \tilde{K}(2 d-t) \int_{d}^{x} \tilde{K}(\xi) \sum_{n=0}^{\infty} \frac{\varphi_{0}\left(t, k_{n}\right) \varphi_{0}\left(\xi, k_{n}\right)}{\alpha_{n}} d \xi d t \\
& -\left(a^{-}\right)^{2} \int_{0}^{x} \tilde{K}(2 d-t) \int_{d}^{x} \tilde{K}(\xi) \sum_{n=0}^{\infty} \frac{\varphi_{0}\left(t, k_{n}\right) \varphi_{0}\left(2 d-\xi, k_{n}\right)}{\alpha_{n}} d \xi d t \\
& +a^{+} a^{-} \int_{0}^{x} \tilde{K}(t) \int_{d}^{x} \tilde{K}(\xi) \sum_{n=0}^{\infty} \frac{\varphi_{0}\left(t, k_{n}^{0}\right) \varphi_{0}\left(2 d-\xi, k_{n}^{0}\right)}{\alpha_{n}^{0}} d \xi d t \\
& +a^{+} a^{-} \int_{0}^{x} \tilde{K}(2 d-t) \int_{d}^{x} \tilde{K}(\xi) \sum_{n=0}^{\infty} \frac{\varphi_{0}\left(t, k_{n}^{0}\right) \varphi_{0}\left(\xi, k_{n}^{0}\right)}{\alpha_{n}^{0}} d \xi d t \\
& -\left(a^{-}\right)^{2} \int_{0}^{x} \tilde{K}(2 d-t) \int_{d}^{x} \tilde{K}(\xi) \sum_{n=0}^{\infty} \frac{\varphi_{0}\left(t, k_{n}^{0}\right) \varphi_{0}\left(2 d-\xi, k_{n}^{0}\right)}{\alpha_{n}^{0}} d \xi d t \\
& -\left(a^{+}\right)^{2} \int_{0}^{x} \tilde{K}(t) \int_{d}^{x} \tilde{K}(\xi) \sum_{n=0}^{\infty} \frac{\varphi_{0}\left(t, k_{n}^{0}\right) \varphi_{0}\left(\xi, k_{n}^{0}\right)}{\alpha_{n}^{0}} d \xi d t=0 .
\end{aligned}
$$

Replacing $2 d-\xi$ by $\xi$ in this equation, we get

$$
\begin{aligned}
& \int_{0}^{x}\left(a^{+} \tilde{K}(t)-a^{-} \tilde{K}(2 d-t)\right)^{2} d t+\sum_{n=0}^{\infty} \frac{1}{\alpha_{n}}\left[\int_{0}^{x}\left(a^{+} \tilde{K}(t)-a^{-} \tilde{K}(2 d-t)\right) \varphi_{0}\left(t, k_{n}\right) d t\right]^{2} \\
& =\sum_{n=0}^{\infty} \frac{1}{\alpha_{n}^{0}}\left[\int_{0}^{x}\left(a^{+} \tilde{K}(t)-a^{-} \tilde{K}(2 d-t)\right) \varphi_{0}\left(t, k_{n}^{0}\right) d t\right]^{2} .
\end{aligned}
$$

Using Parseval's equality

$\int_{0}^{x}\left(a^{+} \tilde{K}(t)-a^{-} \tilde{K}(2 d-t)\right)^{2} d t=\sum_{n=0}^{\infty} \frac{1}{\alpha_{n}^{0}}\left[\int_{0}^{x}\left(a^{+} \tilde{K}(t)-a^{-} \tilde{K}(2 d-t)\right) \varphi_{0}\left(t, k_{n}^{0}\right) d t\right]^{2}$ for the function $a^{+} \tilde{K}(t)-a^{-} \tilde{K}(2 d-t) \in L_{2}(0, \pi)$, extended by zero for $t>x$ we obtain that

$\sum_{n=0}^{\infty} \frac{1}{\alpha_{n}}\left[\int_{0}^{x}\left(a^{+} \tilde{K}(t)-a^{-} \tilde{K}(2 d-t)\right) \varphi_{0}\left(t, k_{n}\right) d t\right]^{2}=0$.

Since $\alpha_{n}>0$ then $\int_{0}^{x}\left(a^{+} \tilde{K}(t)-a^{-} \tilde{K}(2 d-t)\right) \varphi_{0}\left(t, k_{n}\right) d t=0, n=0,1, \ldots$ The system of functions 
$\left\{\varphi_{0}\left(t, k_{n}\right)\right\}_{n \geq 0}$ is complete in $L_{2}(0, \pi)[11]$, we obtain $a^{+} \tilde{K}(t)-a^{-} \tilde{K}(2 d-t)=0$.

That is, $\left(L_{x} \tilde{K}\right)(t)=0$, where $L_{x}$ is the operator which defined above. Since $L_{x}$ has a bounded inverse, we have $\tilde{K}(x, \cdot)=0$. Which means that the main integral equation has a solution and it is unique.

Lemma 2. Assume that numbers $\left\{\lambda_{n}, \alpha_{n}\right\}_{n \geq 0}$ satisfying the conditions of the form(2.4) and (2.5) are given and denote

$$
b(x):=\sum_{n=0}^{\infty}\left(\frac{\cos k_{n} x}{\alpha_{n}}-\frac{\cos k_{n}^{0} x}{\alpha_{n}^{0}}\right)
$$

where $\alpha_{n}^{0}=\left\{\begin{array}{l}\frac{\pi}{2}, n>0 \\ \pi, n=0\end{array}\right.$. Then $b(x) \in W_{2}^{1}(0, d) \cup(d, 2 \pi)$.

Proof. Denote $\varepsilon_{n}=k_{n}-k_{n}^{0}$.

Since $\frac{\cos k_{n} x}{\alpha_{n}}-\frac{\cos k_{n}^{0} x}{\alpha_{n}^{0}}=\frac{1}{\alpha_{n}^{0}}\left(\cos k_{n} x-\cos k_{n}^{0} x\right)+\left(\frac{1}{\alpha_{n}}-\frac{1}{\alpha_{n}^{0}}\right) \cos k_{n} x$,

$\cos k_{n} x-\cos k_{n}^{0} x=-\varepsilon_{n} \sin k_{n}^{0} x-\sin k_{n}^{0} x\left(\sin \varepsilon_{n} x-\varepsilon_{n} x\right)-2 \sin ^{2} \frac{\varepsilon_{n} x}{2} \cos k_{n}^{0} x$

we have $b(x)=B_{1}(x)+B_{2}(x)$ where

$$
\begin{gathered}
B_{1}(x)=-\sum_{n=1}^{\infty} \frac{d_{n} x \sin k_{n}^{0} x}{\alpha_{n}^{0} k_{n}^{0}} \\
B_{2}(x)=\sum_{n=0}^{\infty}\left(\frac{1}{\alpha_{n}}-\frac{1}{\alpha_{n}^{0}}\right) \cos k_{n} x-\sum_{n=1}^{\infty} \frac{\delta_{n} x \sin k_{n}^{0} x}{\alpha_{n}^{0} k_{n}^{0}} \\
-\sum_{n=1}^{\infty}\left(\sin \varepsilon_{n} x-\varepsilon_{n} x\right) \frac{\sin k_{n}^{0} x}{\alpha_{n}^{0}}-\sum_{n=1}^{\infty} 2 \sin ^{2} \frac{\varepsilon_{n} x}{2} \frac{\cos k_{n}^{0} x}{\alpha_{n}^{0}} .
\end{gathered}
$$

Since $\varepsilon_{n}=O\left(\frac{1}{n}\right), \frac{1}{\alpha_{n}}-\frac{1}{\alpha_{n}^{0}}=-\frac{\delta_{n}}{k_{n}^{0}}+O\left(\frac{1}{n}\right)$ where $\delta_{n}=\frac{1}{k_{n}^{0}} \int_{0}^{\pi} K_{t}^{\prime}(\pi, t) \sin k_{n}^{0} t d t$ the series in (3.11) and (3.12) converge absolutely and uniformly on $[0,2 \pi]$ and $B_{2}(x) \in W_{2}^{1}(0, d) \cup(d, 2 \pi)$, $B_{1}(x) \in W_{2}^{1}(0, d) \cup(d, 2 \pi)$.

Consequently $b(x) \in W_{2}^{1}(0, d) \cup(d, 2 \pi)$.

Since $F_{0}(x, t)=\frac{a^{+}}{2}[b(x+t)+b(x-t)]+\frac{a^{-}}{2}[b(x-2 d+t)+b(x+2 d-t)]$, then Lemma 6 implies that $F_{0}(x, t)$ is continuous and $F(x, t)=a^{+} F_{0}(x, t)+a^{-} F_{0}(2 d-x, t) \in W_{2}^{1}(0, \pi)$.

According to (3.3) and (3.4)

$$
\begin{gathered}
F_{0_{t t}}^{\prime \prime}(x, t)=F_{0_{x x}}^{\prime \prime}(x, t), \quad F_{t t}^{\prime \prime}(x, t)=F_{x x}^{\prime \prime}(x, t) \\
\left.F_{0}(x, t)\right|_{x=0}=a^{+} b(t)+a^{-} b(2 d-t), \\
\left.F_{0}(x, t)\right|_{t=0}=a^{+} b(x)+a^{-} \frac{b(2 d+x)+b(2 d-x)}{2}
\end{gathered}
$$




$$
\begin{gathered}
\frac{\partial F_{0}(x, t)}{\partial x}=\left.\frac{\partial F_{0}(\xi, t)}{\partial \xi}\right|_{\xi=x}, \quad \frac{\partial F_{0}(2 d-x, t)}{\partial x}=-\left.\frac{\partial F_{0}(\xi, t)}{\partial \xi}\right|_{\xi=2 d-x} \\
\frac{a^{-}}{a^{+}} \frac{d \tilde{K}(x, x)}{d x}=\frac{d}{d x}[\tilde{K}(x, 2 d-x+0)-\tilde{K}(x, 2 d-x-0)] . \\
\left.\frac{\partial \tilde{K}(x, t)}{\partial t}\right|_{t=0}=0 \\
q(x)=\frac{2}{a^{+}} \frac{d \tilde{K}(x, x)}{d x} .
\end{gathered}
$$

Lemma 3. The following relations hold

$$
\begin{gathered}
-\varphi^{\prime \prime}(x, \lambda)+q(x) \varphi(x, \lambda)=\lambda \varphi(x, \lambda), \quad \lambda=k^{2} \\
\varphi(0, \lambda)=1, \quad \varphi^{\prime}(0, \lambda)=0 .
\end{gathered}
$$

Proof. 1) First we assume that $b(x) \in W_{2}^{2}(0,2 \pi)$ where $b(x)$ is defined by (3.10). Differentiating the identity

$$
J(x, t):=F(x, t)+a^{+} \tilde{K}(x, t)-a^{-} \tilde{K}(x, 2 d-t)+\int_{0}^{x} \tilde{K}(x, \xi) F_{0}(\xi, t) d \xi=0,
$$

we calculate

$$
\begin{aligned}
& J_{t}^{\prime}(x, t)=F_{t}^{\prime}(x, t)+a^{+} \tilde{K}_{t}^{\prime}(x, t)-a^{-} \tilde{K}_{t}^{\prime}(x, 2 d-t)+\int_{0}^{x} \tilde{K}(x, \xi) F_{0_{t}}^{\prime}(\xi, t) d \xi=0, \\
& J_{t t}^{\prime \prime}(x, t)=F_{t t}^{\prime \prime}(x, t)+a^{+} \tilde{K}_{t t}^{\prime \prime}(x, t)-a^{-} \tilde{K}_{t t}^{\prime \prime}(x, 2 d-t) \\
& -\left.[\tilde{K}(x, 2 d-x+0)-\tilde{K}(x, 2 d-x-0)] \frac{\partial F_{0}(\xi, t)}{\partial \xi}\right|_{\xi=2 d-x} \\
& +\left.\tilde{K}(x, x) \frac{\partial F_{0}(\xi, t)}{\partial \xi}\right|_{\xi=x}-\left.\tilde{K}(x, 0) \frac{\partial F_{0}(\xi, t)}{\partial \xi}\right|_{\xi=x}+\left.\frac{\partial \tilde{K}(x, \xi)}{\partial \xi}\right|_{\xi=0} F_{0}(0, t) \\
& -\left.\frac{\partial \tilde{K}(x, \xi)}{\partial \xi}\right|_{\xi=x} F_{0}(x, t)+\left.\frac{\partial \tilde{K}(x, \xi)}{\partial \xi}\right|_{\xi=2 d-x+0} F_{0}(2 d-x+0, t) \\
& -\left.\frac{\partial \tilde{K}(x, \xi)}{\partial \xi}\right|_{\xi=2 d-x-0} ^{\xi=x} F_{0}(2 d-x-0, t)+\int_{0}^{x} \tilde{K}_{\xi \xi}^{\prime \prime}(x, \xi) F_{0}(\xi, t) d \xi=0 \\
& J_{x}^{\prime}(x, t)=F_{x}^{\prime}(x, t)+a^{+} \tilde{K}_{x}^{\prime}(x, t)-a^{-} \tilde{K}_{x}^{\prime}(x, 2 d-t)+\int_{0}^{x} \tilde{K}(x, \xi) F_{0_{x}}^{\prime}(\xi, t) d \xi \\
& \tilde{K}(x, x) F_{0}(x, t)+[\tilde{K}(x, 2 d-x+0)-\tilde{K}(x, 2 d-x-0)] F_{0}(2 d-x, t)=0 \\
& J_{x x}^{\prime \prime}(x, t)=F_{x x}^{\prime \prime}(x, t)+a^{+} \tilde{K}_{x x}^{\prime \prime}(x, t)-a^{-} \tilde{K}_{x x}^{\prime \prime}(x, 2 d-t) \\
& +\int_{0}^{x} \tilde{K}_{x x}^{\prime \prime}(x, \xi) F_{0}(\xi, t) d \xi+\left.\frac{\partial \tilde{K}(x, \xi)}{\partial x}\right|_{\xi=2 d-x+0} F_{0}(2 d-x+0, t) \\
& -\left.\frac{\partial \tilde{K}(x, \xi)}{\partial x}\right|_{\xi=2 d-x-0} F_{0}(2 d-x-0, t)+\left.\frac{\partial \tilde{K}(x, \xi)}{\partial x}\right|_{\xi=x} F_{0}(x, t) \\
& +\frac{d \tilde{K}(x, x)}{d x} F_{0}(x, t)+\tilde{K}(x, x) \frac{\partial F_{0}(x, t)}{\partial x} \\
& +[\tilde{K}(x, 2 d-x+0)-\tilde{K}(x, 2 d-x-0)] \frac{\partial F_{0}(2 d-x, t)}{\partial x} \\
& +\frac{d}{d x}[\tilde{K}(x, 2 d-x+0)-\tilde{K}(x, 2 d-x-0)] F_{0}(2 d-x, t)=0 .
\end{aligned}
$$


It follows from (3.21), (3.23) and (3.24) and the equality

$$
J_{x x}^{\prime \prime}(x, t)-J_{t t}^{\prime \prime}(x, t)-q(x) J(x, t) \equiv 0
$$

that

$$
\begin{gathered}
a^{+} \tilde{K}_{x x}^{\prime \prime}(x, t)-a^{-} \tilde{K}_{x x}^{\prime \prime}(x, 2 d-t)-\left[a^{+} \tilde{K}_{t t}^{\prime \prime}(x, t)-a^{-} \tilde{K}_{t t}^{\prime \prime}(x, 2 d-t)\right] \\
\quad-q(x)\left[\tilde{K}(x, t)-a^{-} \tilde{K}(x, 2 d-t)\right] \\
+\int_{0}^{x}\left[\tilde{K}_{x x}^{\prime \prime}(x, \xi)-\tilde{K}_{t t}^{\prime \prime}(x, \xi)-q(x) \tilde{K}(x, \xi)\right] F_{0}(\xi, t) d \xi=0 .
\end{gathered}
$$

According to Theorem 4 , this homogeneous equation has only the trivial solution, i.e.

$$
\tilde{K}_{x x}^{\prime \prime}(x, t)-\tilde{K}_{t t}^{\prime \prime}(x, t)-q(x) \tilde{K}(x, t)=0, \quad 0<t<x .
$$

Differentiating (3.5) twice, we get

$$
\begin{aligned}
& \varphi^{\prime}(x, \lambda)=\varphi_{0}^{\prime}(x, \lambda)+\int_{0}^{x} \tilde{K}_{x}^{\prime}(x, t) \cos k t d t+\tilde{K}(x, x) \cos k x \\
&+ {[\tilde{K}(x, 2 d-x+0)-\tilde{K}(x, 2 d-x-0)] \cos k(2 d-x) } \\
& \varphi^{\prime \prime}(x, \lambda)=\varphi_{0}^{\prime \prime}(x, \lambda)+\int_{0}^{x} \tilde{K}_{x x}^{\prime \prime}(x, t) \cos k t d t \\
&+\left.\frac{\partial \tilde{K}(x, t)}{\partial x}\right|_{t=x} \cos k x+\frac{d \tilde{K}(x, x)}{d x} \cos k x-\tilde{K}(x, x) k \sin k x \\
&+\left[\left.\frac{\partial \tilde{K}(x, t)}{\partial x}\right|_{t=2 d-x+0}-\left.\frac{\partial \tilde{K}(x, t)}{\partial x}\right|_{t=2 d-x-0}\right] \cos k(2 d-x) \\
&+\frac{d}{d x}[\tilde{K}(x, 2 d-x+0)-\tilde{K}(x, 2 d-x-0)] \cos k(2 d-x) \\
&+[\tilde{K}(x, 2 d-x+0)-\tilde{K}(x, 2 d-x-0)] k \sin k(2 d-x) .
\end{aligned}
$$

On the other hand, integrating by parts twice, we obtain

$$
\begin{aligned}
\lambda \varphi(x, \lambda) & =k^{2} \varphi_{0}(x, \lambda) \\
- & {[\tilde{K}(x, 2 d-x+0)-\tilde{K}(x, 2 d-x-0)] k \sin k(2 d-x) } \\
+ & \tilde{K}(x, x) k \sin k x-\left.\frac{\partial \tilde{K}(x, t)}{\partial t}\right|_{t=0}+\left.\frac{\partial \tilde{K}(x, t)}{\partial t}\right|_{t=x} \cos k x \\
- & {\left[\left.\frac{\partial \tilde{K}(x, t)}{\partial t}\right|_{t=2 d-x+0}-\left.\frac{\partial \tilde{K}(x, t)}{\partial t}\right|_{t=2 d-x-0} \cos k(2 d-x)\right.} \\
& -\int_{0}^{x} \frac{\partial^{2} \tilde{K}(x, t)}{\partial t^{2}} \cos k t d t .
\end{aligned}
$$

Together with (3.5) and (3.28) this gives

$$
\begin{aligned}
& \varphi^{\prime \prime}(x, \lambda)+\lambda \varphi(x, \lambda)-q(x) \varphi(x, \lambda)=\left[\left.\frac{\partial \tilde{K}(x, t)}{\partial x}\right|_{t=x}+\left.\frac{\partial \tilde{K}(x, t)}{\partial t}\right|_{t=x}\right] \cos k x \\
& +\frac{d \tilde{K}(x, x)}{d x} \cos k x+\left[\left.\frac{\partial \tilde{K}(x, t)}{\partial x}\right|_{t=2 d-x+0}-\left.\frac{\partial \tilde{K}(x, t)}{\partial t}\right|_{t=2 d-x+0} \cos k(2 d-x)\right. \\
& -\left[\left.\frac{\partial \tilde{K}(x, t)}{\partial x}\right|_{t=2 d-x-0}-\left.\frac{\partial \tilde{K}(x, t)}{\partial t}\right|_{t=2 d-x-0}\right] \cos k(2 d-x)-\left.\frac{\partial \tilde{K}(x, t)}{\partial t}\right|_{t=0} \\
& +\frac{d}{d x}[\tilde{K}(x, 2 d-x+0)-\tilde{K}(x, 2 d-x-0)] \cos k(2 d-x)-2 \frac{d \tilde{K}(x, x)}{d x} \cos k x \\
& -2 \frac{d}{d x}[\tilde{K}(x, 2 d-x+0)-\tilde{K}(x, 2 d-x-0)] \cos k(2 d-x) \\
& +\int_{0}^{x}\left[\tilde{K} \tilde{x}_{x x}^{\prime \prime}(x, t)-\tilde{K}_{t t}^{\prime \prime}(x, t)-q(x) \tilde{K}(x, t)\right] \cos k t d t .
\end{aligned}
$$

Taking (3.16), (3.18) and (3.26) into account, we arrive at (3.19). The relations (3.20) follow from (3.5) and (3.27) for $x=0$. 
Lemma 4. For each function $g(x) \in L_{2}(0, \pi)$

$$
\int_{0}^{\pi} g^{2}(x) d x=\sum_{n=1}^{\infty} \frac{1}{\alpha_{n}}\left(\int_{0}^{\pi} g(t) \varphi\left(t, k_{n}\right) d t\right)^{2} .
$$

Proof of this Lemma can be done by using proof of Lemma in reference [2].

Corollary 1. For arbitrary functions $f(x), g(x) \in L_{2}(0, \pi)$

$$
\int_{0}^{\pi} f(x) g(x) d x=\sum_{n=0}^{\infty} \frac{1}{\alpha_{n}} \int_{0}^{\pi} f(t) \varphi\left(t, k_{n}\right) d t \int_{0}^{\pi} g(t) \varphi\left(t, k_{n}\right) d t
$$

Lemma 5. The following relation holds

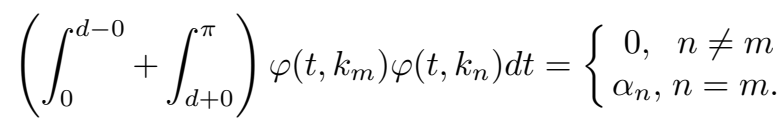

Proof. 1) Let $f(x) \in W_{2}^{2}[0, \pi]$. Consider the series

$$
f^{*}(x)=\sum_{n=0}^{\infty} c_{n} \varphi\left(x, k_{n}\right)
$$

where

$$
c_{n}:=\frac{1}{\alpha_{n}} \int_{0}^{\pi} f(x) \varphi\left(x, k_{n}\right) d x .
$$

By using Lemma 5 and integration by parts we obtain

$$
\begin{aligned}
c_{n}= & \frac{1}{\alpha_{n} k_{n}} \int_{0}^{\pi} f(x)\left[-\varphi^{\prime \prime}\left(x, k_{n}\right)+q(x) \varphi\left(x, k_{n}\right)\right] d x \\
& =-\frac{1}{\alpha_{n} k_{n}} f(\pi) \varphi^{\prime}\left(\pi, k_{n}\right)+\frac{1}{\alpha_{n} k_{n}} f(0) \varphi^{\prime}\left(0, k_{n}\right) \\
& +\frac{1}{\alpha_{n} k_{n}} f^{\prime}(\pi) \varphi\left(\pi, k_{n}\right)-\frac{1}{\alpha_{n} k_{n}} f^{\prime}(0) \varphi\left(0, k_{n}\right) \\
& +\frac{1}{\alpha_{n} k_{n}} \int_{0}^{\pi} \varphi\left(x, k_{n}\right)\left[-f^{\prime \prime}(x)+q(x) f(x)\right] d x
\end{aligned}
$$

Applying the asymptotic formulae, (2.4) and (2.5) one can check that for $n \rightarrow \infty$

$$
c_{n}=O\left(\frac{1}{n^{2}}\right), \varphi\left(x, k_{n}\right)=O(1)
$$

uniformly for $x \in[0, \pi]$. Therefore the series (3.33) converge absolutely and uniformly on $[0, \pi]$. According to (3.31) and (3.34)

$\int_{0}^{\pi} f(x) g(x) d x=\sum_{n=0}^{\infty} c_{n} \int_{0}^{\pi} g(t) \varphi\left(t, k_{n}\right) d t=\int_{0}^{\pi} g(t) \sum_{n=0}^{\infty} c_{n} \varphi\left(t, k_{n}\right) d t=\int_{0}^{\pi} g(t) f^{*}(t) d t$.

Since $g(x)$ is arbitrary, we obtain $f^{*}(x)=f(x)$, i.e.

$$
f(x)=\sum_{n=0}^{\infty} c_{n} \varphi\left(x, k_{n}\right) .
$$

2) Fix $m \geq 0$ and take $f(x)=\varphi\left(x, k_{m}\right)$. Then, by virtue of (3.35) 


$$
\varphi\left(x, k_{m}\right)=\sum_{n=0}^{\infty} c_{n m} \varphi\left(x, k_{n}\right), \quad c_{n m}=\frac{1}{\alpha_{n}} \int_{0}^{\pi} \varphi\left(x, k_{m}\right) \varphi\left(x, k_{n}\right) d x .
$$

Further, the system $\left\{\varphi\left(x, k_{n}\right)\right\}_{n>0}$ is minimal in $L_{2}(0, \pi)$ and consequently, in view of (3.5), the system $\left\{\varphi\left(x, k_{n}\right)\right\}_{n \geq 0}$ is also minimal in $L_{2}(0, \pi)$. Hence $c_{n m}=\delta_{n m}\left(\delta_{n m}\right.$ is the Kronecker delta), and we arrive at $(3.32)$.

Lemma 6. The constants $a$ and $d$ in the eigenvalue problem (1.1)-(1.3) are uniquely determined by the eigenvalues provided $0<d<\frac{\pi}{2}$ and $|a-1|>0$.

Proof. The proof is similar with Lemma 5 [10, Lemma 6].

Lemma 7. For all $n \geq 0$,

$$
\varphi\left(\pi, k_{n}\right)=0
$$

$$
\varphi\left(d+0, k_{n}\right)=a \varphi\left(d-0, k_{n}\right), \quad \varphi^{\prime}\left(d+0, k_{n}\right)=a^{-1} \varphi^{\prime}\left(d-0, k_{n}\right)
$$

Proof. It follows, from Lemma 2 in [3]

$$
\left(k_{n}-k_{m}\right) \int_{0}^{\pi} \varphi\left(x, k_{m}\right) \varphi\left(x, k_{n}\right) d x=\left(\varphi^{\prime}\left(x, k_{m}\right) \varphi\left(x, k_{n}\right)-\varphi\left(x, k_{m}\right) \varphi^{\prime}\left(x, k_{n}\right)\right)\left(\left.\right|_{0} ^{d-0}+\left.\right|_{d+0} ^{\pi}\right) .
$$

Taking (3.32) into account, we get

$$
\varphi^{\prime}\left(\pi, k_{m}\right) \varphi\left(\pi, k_{n}\right)-\varphi\left(\pi, k_{m}\right) \varphi^{\prime}\left(\pi, k_{n}\right)=0 .
$$

Clearly, $\varphi^{\prime}\left(\pi, k_{n}\right) \neq 0$ for all $n \geq 0$. Indeed, if we suppose that $\varphi^{\prime}\left(\pi, k_{m}\right)=0$ for a certain $m$, then $\varphi^{\prime}\left(\pi, k_{n}\right)=0$ and in view of (3.37) for $n \neq m$. From (3.5)

$$
\varphi^{\prime}\left(\pi, k_{n}\right)=\varphi_{0}^{\prime}\left(\pi, k_{n}\right)+\int_{0}^{\pi} \tilde{K}_{x}(\pi, t) \cos k_{n} t d t+\tilde{K}(\pi, \pi) \cos k_{n} \pi
$$

i.e. $\varphi^{\prime}\left(\pi, k_{n}\right) \sim \varphi_{0}^{\prime}\left(\pi, k_{n}\right) \neq 0$ for $n \rightarrow \infty$, it contradicts with $\varphi^{\prime}\left(\pi, k_{n}\right)=0$ for $n \neq m$.

Thus $\varphi^{\prime}\left(\pi, k_{n}\right) \neq 0$ for all $n \geq 0$. Since $\varphi^{\prime}\left(\pi, k_{n}\right) \neq 0$ for all $n \geq 0$, from (3.38) we obtain

$$
\frac{\varphi\left(\pi, k_{n}\right)}{\varphi^{\prime}\left(\pi, k_{n}\right)}=\frac{\varphi\left(\pi, k_{m}\right)}{\varphi^{\prime}\left(\pi, k_{m}\right)}=H
$$

That is, $\varphi^{\prime}\left(\pi, k_{n}\right) H=\varphi\left(\pi, k_{n}\right)$ for all $n \geq 0$. Since $\varphi\left(\pi, k_{n}\right)=o(1)$ for $n \rightarrow \infty$, we obtain $H=0$. Hence $\varphi\left(\pi, k_{n}\right)=0$ for all $n \geq 0$.

From (3.5) for $x \rightarrow d+0, \varphi\left(d+0, k_{n}\right)-a \varphi\left(d-0, k_{n}\right)=0$.

Since $\varphi^{\prime}(x, \lambda)=\varphi_{0}^{\prime}(x, \lambda)+\tilde{K}(x, x) \cos k x+\int_{0}^{x} \tilde{K}_{x}(x, t) \cos k t d t$,

$\varphi^{\prime}\left(d+0, k_{n}\right)-a^{-1} \varphi^{\prime}\left(d-0, k_{n}\right)=0$.

Together with Lemma 4, Lemma 6 and Lemma 7 this gives that the numbers $\left\{\lambda_{n}, \alpha_{n}\right\}_{n>0}$ are the spectral data for the constructed boundary value problem $L(q(x))$. Thus, the following Theorem is proved.

Theorem 5. For real numbers $\left\{\lambda_{n}, \alpha_{n}\right\}_{n>0}$ to be spectral data for a certain boundary value problem $L(q(x))$ with $q(x) \in L_{2}(0, \pi)$, it is necessary and sufficient that $k_{n} \neq k_{m}(n \neq m), \alpha_{n}>0$, and that (2.4)-(2.5) hold.

The boundary value problem $L(q(x))$ can be constructed by the following algorithm: 
Algorithm 1. (i) From the given numbers $\left\{\lambda_{n}, \alpha_{n}\right\}_{n>0}$ construct the function $F(x, t)$ by (3.4).

(ii) Find the function $K(x, t)$ by solving equation (3.6).

(iii) Calculate $q(x)$ by the formulae (3.18).

Example 1. Assume that the spectral data of some eigenvalue problem of the form (1.1)-(1.3) is the following:

$k_{n}=k_{n}^{0}, \quad \alpha_{n}=\alpha_{n}^{0}+\delta_{n}, \quad k_{n}^{0}=n+h_{n}, \quad h_{n} \in \ell_{2}$.

Since $h_{n} \in(0,1)$ for all $n \in N, h_{n}=\frac{1}{2}$. Let $k_{n}^{0}=n+\frac{1}{2}, d=\frac{\pi}{2}$.

Then from (3.3) and (3.4), we have

$$
\begin{gathered}
F_{0}(x, t)=\left(\frac{1}{\alpha_{0}}-\frac{1}{\alpha_{0}^{0}}\right)\left(a^{+} \cos \frac{x}{2} \cos \frac{t}{2}+a^{-} \cos \frac{x}{2} \sin \frac{t}{2}\right) \\
F(x, t)=\left(\frac{1}{\alpha_{0}}-\frac{1}{\alpha_{0}^{0}}\right)\left[\frac{\left(a^{+}\right)^{2}}{2} \cos \left(\frac{x-t}{2}\right)+a \cdot a^{-} \sin \frac{x+t}{2}-\sin \frac{x}{2} \sin \frac{t}{2}\right] .
\end{gathered}
$$

Solving equation (3.6) and by using relation (3.18) we obtain

$$
\widetilde{K}(x, t)=-\left(\frac{1}{\alpha_{0}}-\frac{1}{\alpha_{0}^{0}}\right) \frac{\left(a^{+} \cos \frac{x}{2}+a^{-} \sin \frac{x}{2}\right)\left(\left[\left(a^{+}\right)^{2}+\left(a^{-}\right)^{2}\right] \cos \frac{t}{2}+2 a^{+} a^{-} \sin \frac{t}{2}\right)}{1+\left(\frac{1}{\alpha_{0}}-\frac{1}{\alpha_{0}^{0}}\right)\left[\frac{\left(a^{+}\right)^{2}+\left(a^{-}\right)^{2}}{2}(x+\sin x)+2 a^{+} a^{-} \sin \frac{x}{2}\right]}
$$

and

$$
\begin{aligned}
& q(x)=-\frac{1}{a^{+}}\left(\frac{1}{\alpha_{0}}-\frac{1}{\alpha_{0}^{0}}\right) \frac{-\frac{a^{+}}{2}\left(\frac{1}{\alpha_{0}}-\frac{1}{\alpha_{0}^{0}}\right)\left[\left(a^{+}\right)^{2}+3\left(a^{-}\right)^{2}\right]\left[1+\left(a^{+}\right)^{2}+3\left(a^{-}\right)^{2}\right]}{\left(1+\left(\frac{1}{\alpha_{0}}-\frac{1}{\alpha_{0}^{0}}\right)\left[\frac{\left(a^{+}\right)^{2}+\left(a^{-}\right)^{2}}{2}(x+\sin x)+2 a^{+} a^{-} \sin \frac{x}{2}\right]\right)^{2}} \\
& \frac{+\left(-a^{+}-\frac{a^{+}}{2}\left(\frac{1}{\alpha_{0}}-\frac{1}{\alpha_{0}^{0}}\right)\left(\left(a^{+}\right)^{2}+\left(a^{-}\right)^{2}\right)\left[x-\left(3\left(a^{+}\right)^{2}+\left(a^{-}\right)^{2}\right)\right]\right) \sin x}{\left(1+\left(\frac{1}{\alpha_{0}}-\frac{1}{\alpha_{0}^{0}}\right)\left[\frac{\left(a^{+}\right)^{2}+\left(a^{-}\right)^{2}}{2}(x+\sin x)+2 a^{+} a^{-} \sin \frac{x}{2}\right]\right)^{2}} \\
& +\left(\frac{1}{\alpha_{0}}-\frac{1}{\alpha_{0}^{0}}\right)\left(\left(a^{+}\right)^{2}+\left(a^{-}\right)^{2}\right)\left[\frac{a^{-}}{2}\left(3\left(a^{+}\right)^{2}+\left(a^{-}\right)^{2}\right) x-\frac{a^{+}}{2}\left(1+3\left(a^{+}\right)^{2}+\left(a^{-}\right)^{2}\right)\right] \cos x \\
& \frac{\left.+a^{-}\left(3\left(a^{+}\right)^{2}+\left(a^{-}\right)^{2}\right) \cos x+2 a^{+} a^{-}\left(\frac{1}{\alpha_{0}^{0}}\right)\left[\frac{\left(a^{+}\right)^{2}+\left(a^{-}\right)^{2}}{2}(x+\sin x)+2 a^{+} a^{-} \sin \frac{x}{2}\right]\right)^{2}\left[-a^{+} \sin x+a^{-}\left(3\left(a^{+}\right)^{2}+\left(a^{-}\right)^{2}\right) \cos x\right] \sin \frac{x}{2}}{\left(1+\left(\frac{1}{\alpha_{0}}-\frac{1}{\alpha_{0}^{0}}\right)\left[\frac{\left(a^{+}\right)^{2}+\left(a^{-}\right)^{2}}{2}(x+\sin x)+2 a^{+} a^{-} \sin \frac{x}{2}\right]\right)^{2}} \\
& +a^{+} a^{-}\left(\frac{1}{\alpha_{0}}-\frac{1}{\alpha_{0}^{0}}\right)\left[-a^{+}\left(1+\left(a^{+}\right)^{2}+3\left(a^{-}\right)^{2}\right)-a^{-}\left(3\left(a^{+}\right)^{2}+\left(a^{-}\right)^{2}\right) \sin x\right] \cos \frac{x}{2} \\
& \left(1+\left(\frac{1}{\alpha_{0}}-\frac{1}{\alpha_{0}^{0}}\right)\left[\frac{\left(a^{+}\right)^{2}+\left(a^{-}\right)^{2}}{2}(x+\sin x)+2 a^{+} a^{-} \sin \frac{x}{2}\right]\right)^{2}
\end{aligned}
$$


In order to reconstruct the second boundary, we construct the solution $\varphi(x, \lambda)$ by using $(3.15)$

$$
\begin{aligned}
& \varphi(x, \lambda)=\varphi_{0}(x, \lambda) \\
& -\left(\frac{1}{\alpha_{0}}-\frac{1}{\alpha_{0}^{0}}\right) \frac{4 k \sin k x\left[\left(\left(a^{+}\right)^{2}+\left(a^{-}\right)^{2}\right)\left(a^{+} \cos x+a^{-} \sin x\right)\right]}{\left(4 k^{2}-1\right)\left(1+\left(\frac{1}{\alpha_{0}}-\frac{1}{\alpha_{0}^{0}}\right)\left[\frac{\left(a^{+}\right)^{2}+\left(a^{-}\right)^{2}}{2}(x+\sin x)+2 a^{+} a^{-} \sin \frac{x}{2}\right]\right)} \\
& \frac{+4 k \sin k x\left[a^{+}\left(\left(a^{+}\right)^{2}+3\left(a^{-}\right)^{2}\right)+2 a^{+} a^{-}\left(a^{+} \sin x-a^{-} \cos x\right)\right]}{\left(4 k^{2}-1\right)\left(1+\left(\frac{1}{\alpha_{0}}-\frac{1}{\alpha_{0}^{0}}\right)\left[\frac{\left(a^{+}\right)^{2}+\left(a^{-}\right)^{2}}{2}(x+\sin x)+2 a^{+} a^{-} \sin \frac{x}{2}\right]\right)} \\
& \frac{+2 \cos k x\left[\left(\left(a^{+}\right)^{2}+\left(a^{-}\right)^{2}\right)\left(a^{-} \cos x-a^{+} \sin x\right)+a^{-}\left(\left(a^{+}\right)^{2}-\left(a^{-}\right)^{2}\right)\right]}{\left(4 k^{2}-1\right)\left(1+\left(\frac{1}{\alpha_{0}}-\frac{1}{\alpha_{0}^{0}}\right)\left[\frac{\left(a^{+}\right)^{2}+\left(a^{-}\right)^{2}}{2}(x+\sin x)+2 a^{+} a^{-} \sin \frac{x}{2}\right]\right)} \\
& \frac{+4 a^{+} a^{-} \cos k x\left(a^{+} \cos x+a^{-} \sin x\right)}{\left(4 k^{2}-1\right)\left(1+\left(\frac{1}{\alpha_{0}}-\frac{1}{\alpha_{0}^{0}}\right)\left[\frac{\left(a^{+}\right)^{2}+\left(a^{-}\right)^{2}}{2}(x+\sin x)+2 a^{+} a^{-} \sin \frac{x}{2}\right]\right)} .
\end{aligned}
$$

\section{References}

1. Agranovich, Z.S. and Marchenko, V. A., The lnverse Problem Scaterring Theory (New York: Gordonand Breach) (1963).

2. Akhmedova, E.N., Huseynov, H. M., On Inverse Problem Sturm-Liouville Operator with Discontinuous Coefficients, Trnasactions of Saratov University, vol.10, Mathematical, Mechanical and Informatical series no.1, (2010)

3. Amirov, R. Kh., On Sturm-Liouville Operators with Discotinuity conditions inside an interval, J. Math. Anal. Appl. 317 (2006), pp. 163-176.

4. Borg, G., Eine umkehrung der Sturm Liouvillesehen eigenwertaufgabe, Acta Math. 78 (1946), pp. 1-96.

5. Coen, S., On the elastic profiles of a profiles of a layered medium from reflection data. Part I. Plane-wave sources J. Acoust. Soc. Am. 70 (1981), pp. 172-5.

6. Conway, J.B., Functions of One Complex Variable. 2. Edition, vol. I, Springer-Verlag, New York, (1995).

7. Faydaoğlu, Ş., and Guseinov, G. Sh., An expansion result for a Sturm-Liouville eigenvalue problem with impulse, Turk. J. Math. 34, No. 3, (2010), pp. 355-366.

8. Gelfand I.M. and Levitan,B.M., On the determination of a differential equation from its spectral function, Izv. Akad. Nauk SSR. Ser. Mat. 15 (1951), pp.309-360 (in Russian), English transl. in Amer. Math. Soc. Transl. Ser. 2 (1) (1955), pp.253-304.

9. Guseinov, I. M. and Mammadova, L. I., Reconstruction of the Diffusion Equation with Singular Coefficients for Two Spectra, Doklady Mathematies, vol.9, no. 1, (2014), pp. 401-404.

10. Hald, O.H., Discontinuous inverse eigenvalue problem, Commun. Pure Appl. Math. 37 (1984) 539-577.

11. He, X. and Volkmer, H., Riesz bases of Sturm-Liouville equations, J. Fourier Anal. Appl. 7(2001), 297-307.

12. Krueger, R. J., An inverse problem for an absorbing medium with multiple discontinuities Q. Appl. Math. 34 (1976), pp. 129-47.

13. Krueger, R.J., Inverse problems for nonabsorbing media with discontinuous material properties, J. Math. Phys. 23 (1982), pp. 396-404.

14. Levitan, B.M. (1984). Inverse Sturm-Liouville Problems, Moscow: Nauka,, (Engl. Transl.1987 (Utrecth: VNU Science Press)).

15. Litvinenko, O.N. and Soshnikov, V.I., The theory of Heterogeneous Lines and Their Applications in Radio Engineering, Radio, Moscow, (1964) (in Russian).

16. Marchenko, V.A.,Some Problems in the Theory of Second-Order Differential Operators, Dokl. Akad.,Nauk SSSR. $72,(1950)$, pp. 457-560.

17. Marchenko, V.A., Sturm Liouville Operators and Their Applications, Naukova Dumka, Kiev, 1977, English transl.: Birkhäuser, (1986).

18. Meschanov, V.P. and Feldstein, A.L., Automatic Design of Directional Couplers, Sviaz, Moscow, (1980).

19. Newton, R. G., Inversion ofrcflection data for layered media: a review of exact methods Geophys. J. R.Aslron. Soc. 65 (1981), pp.191-215. 
20. Pöschel, J., Trubowitz, E., Inverse Spectral Theory, Academic Press, Orlando, (1987).

21. Yurko, V.A., Boundary value Problems with Discontinuity Conditions in an Interior of the Interval,Differential Equations, vol. 36, No.8, (2000), pp. 1266-1269.

22. Yurko, V.A., Method of Spectral Mappings in the Inverse Problem Theory, in: Inverse Ill-posed Probl. Ser., VSP, Utrecht, (2002). 